\title{
Different Nature-Inspired Techniques Applied for Motion Planning of Wheeled Robot: A Critical Review
}

\author{
Abhishek Kumar Kashyap ${ }^{1}$ and Anish Pandey ${ }^{2 *}$ \\ ${ }^{1,2}$ School of Mechanical Engineering, KIIT University, Bhubaneswar, PIN - 751024, India
}

Received: July 07, 2018; Accepted: July 20, 2018; Published: July 30, 2018

${ }^{*}$ Corresponding author: Anish Pandey, School of Mechanical Engineering, KIIT University, Bhubaneswar, PIN - 751024, India, E-mail: anish06353@gmail.com

\begin{abstract}
Motion planning is one of the most important parts when we design any wheeled or walking robots. The meaning of motion planning is to plan a path from start point to goal point between obstacles in any working environment. Many techniques have applied by researchers from last two-three decades for wheeled robot motion planning. This article presents a critical review on motion planning of wheeled robot by using different nature-inspired techniques like evolutionary algorithm and swarm-based optimization algorithm.
\end{abstract}

Keywords: Motion planning; Wheeled robot; Working environment; Nature-inspired techniques; Evolutionary algorithm;

\section{Introduction}

Navigation is defined as a technology of guiding a wheeled robot by obtaining its location, path to be travelled and distance. Navigation is all about determining an efficient path to the desired location by avoiding collisions, decreasing the travel taken and conserving energy. Wheeled robot navigation is thus a technique in which the robot is guided between obstacles to reach the goal from starting point without any collision with the obstacles. Demand of wheeled robot is expanding continuously as in increase of complexity, advancement of hardware and researches carried out at dangerous places. The necessity of wheeled robot has been increased as it becomes impossible and too dangerous to interact with some situations like the fire, nuclear test, etc. for the human. Therefore, the replacement of human by wheeled robot become necessary to protect lives and to execute dangerous and delegate works.

The aim of the robotic motion planning is to get the optimum path which must be collision-free between the start and goal position. Planning of path is done in such a way that it is broken into line segments between designed sub-objectives or step points. It is designed for the robot to take steps towards the target by following those line segments in the navigation phase. Configuration space usually represents the navigation environment. Determination of optimality criterion for the path is done by taking the surrounding environment and the running conditions into consideration. To figure out the motion planning problem of wheeled robot, the various nature-based optimization techniques have been applied by authors. Naturebased optimization techniques are further classified into the evolutionary algorithm and swarm-based optimization as shown in Figure 1. Similarly, in Table 1, we have summarized some nature-inspired techniques based motion planning of wheeled robot, which were developed by various authors.

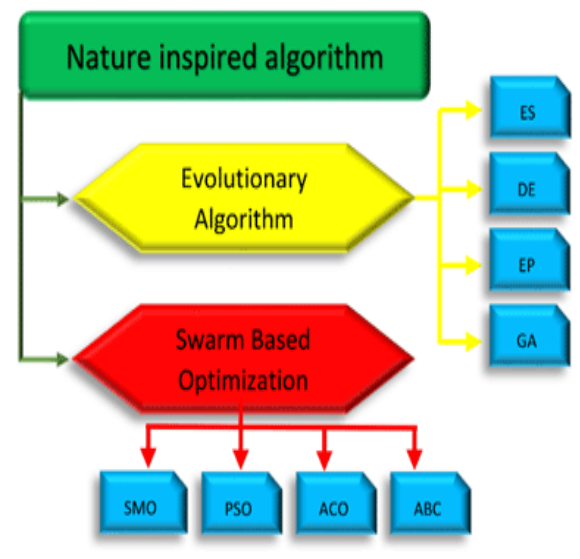

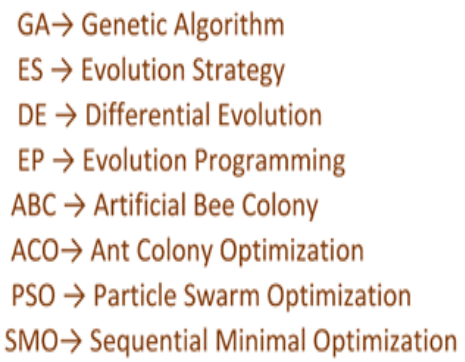

$\mathrm{GA} \rightarrow$ Genetic Algorithm

ES $\rightarrow$ Evolution Strategy

$\mathrm{DE} \rightarrow$ Differential Evolution

$E P \rightarrow$ Evolution Programming

$\mathrm{ABC} \rightarrow$ Artificial Bee Colony

$\mathrm{ACO} \rightarrow$ Ant Colony Optimization

$\mathrm{SMO} \rightarrow$ Sequential Minimal Optimization

Figure 1: Classification of nature-inspired algorithm 
Table 1: Summary of some nature-inspired techniques based motion planning of wheeled robot applied by various authors

\begin{tabular}{|c|c|}
\hline Author(s) & Name of different motion planning techniques applied by authors \\
\hline Chen and Li, [4] & Path planning of a wheeled robot by applying stochastic particle swarm optimization algorithm \\
\hline Huang, [7] & $\begin{array}{l}\text { FPGA (Field Programmable Gate Array)-based path and motion of autonomous robot navigation using particle } \\
\text { swarm optimization algorithm }\end{array}$ \\
\hline Masehian and Sedighizadeh, [10] & Designed multi-objective particle swarm optimization algorithm for robot path planning \\
\hline Mohanta, et al. [19] & $\begin{array}{l}\text { Designed autonomous mobile robot navigation and obstacle avoidance using Petri-Genetic algorithm } \\
\text { optimization }\end{array}$ \\
\hline Castillo, et al. [21] & $\begin{array}{l}\text { Implemented multiple objective genetic algorithms for path-planning optimization of an autonomous wheeled } \\
\text { robots }\end{array}$ \\
\hline Abadi and khooban, [30] & Used Mamdani-type fuzzy logic controller for route tracking of an autonomous wheeled robots \\
\hline Surmann, et al. [31] & Fuzzy system based indoor mobile robot navigation \\
\hline Singh, et al. [33] & Designed intelligent fuzzy controller for mobile robot path planning \\
\hline Abdessemed, et al. [35] & Presented fuzzy based reactive controller for a non-holonomic mobile robot \\
\hline Qing-yong, et al. [37] & Implemented fuzzy behaviour-based architecture for navigation of mobile robot in unknown environments \\
\hline Pratihar, et al. [42] & Navigation of mobile robot among moving obstacles using genetic-fuzzy approach \\
\hline Juang and Chang, [45] & $\begin{array}{l}\text { Wheeled robot navigation in unknown environments using Evolutionary-group-based particle-swarm } \\
\text { optimized fuzzy controller }\end{array}$ \\
\hline Kundu, et al. [47] & Designed navigational strategy for mobile robot using fuzzy-neuro approach \\
\hline Pradhan, et al. [48] & Presented navigation of multiple mobile robots using rule-based-neuro-fuzzy technique \\
\hline Algabri, et al. [50] & $\begin{array}{l}\text { Made a comparative study of soft computing techniques for mobile robot navigation in an unknown } \\
\text { environment }\end{array}$ \\
\hline Lee, [53] & Proposed behaviours based autonomous robot by applying artificial neural network and genetic algorithm \\
\hline Rusu, et al. [55] & Presented behaviour-based neuro-fuzzy controller for wheeled robot navigation \\
\hline Miao and Tian, [67] & Presented dynamic robot path planning using an enhanced simulated annealing approach \\
\hline Chang and Song, [68] & Used Sensor-Based Motion Planning of a Mobile Robot in a Dynamic Environment \\
\hline Liang and $\mathrm{Xu},[73]$ & $\begin{array}{l}\text { Designed genetic algorithm and modified simulated annealing algorithm for global path planning of mobile } \\
\text { robot }\end{array}$ \\
\hline $\mathrm{Bi}$, et al. [81] & Used Ant Colony Algorithm for mobile Robot Navigation in Unknown Dynamic Environment \\
\hline Liang, et al. [82] & Applied Adaptive Bacterial Foraging Algorithm for Mobile Robot Path Planning \\
\hline Hsu, et al. [90] & Presented Path Planning for Mobile Robots Based on Improved Ant Colony Optimization \\
\hline Brand and $\mathrm{Yu},[98]$ & Designed Autonomous Robot Path Optimization using Firefly Algorithm \\
\hline Pandey and Parhi, [100] & $\begin{array}{c}\text { Presented optimum path planning of mobile robot in unknown static and dynamic environments using fuzzy- } \\
\text { wind driven optimization algorithm }\end{array}$ \\
\hline Patle, et al. [102] & Presented Path planning in uncertain environment by using firefly algorithm \\
\hline Elbanhawi and Simic, [104] & Presented a review article on robot motion planning \\
\hline
\end{tabular}

Remaining paper is standardized as follows: Wheeled Robot Motion Planning by The Implementation of Various NatureInspired Techniques Portion describes the literature survey of several nature-inspired techniques used for wheeled robot motion planning. Discussions and Summary Portion discusses the discussions and summary of the current literature survey.

\section{Wheeled Robot Motion Planning by The Implementation of Various Nature-Inspired Techniques}

Researchers have introduced many soft nature-inspired techniques for the wheeled robot motion planning and shunning hindrances problem in several environments in past decades. Taking fitness into consideration, every technique performs their respective works. The implementation of various techniques in wheeled robot motion planning and obstacle avoidance is described below: -

Particle Swarm Optimization Algorithm Encoded in Wheeled Robot

Particle Swarm Optimization (PSO) represents frequently used algorithm for deciding best route because of its directness 
and effectiveness. Kennedy and Ebehart, [1] have proposed this algorithm, but we should also remember the contribution of Heppner and Grenander, [2] for providing the model of biological population. PSO algorithm had become accessible and auspicious approach and had been advanced to get better output for realworld optimization project [3]. Chen and Li, [4] have described the use of PSO approach to execute problem for wheeled robot navigation in terrains having static obstacles.

Thang, et al. [5] have improved PSO for the wheeled robot in predefined working terrains. They generated active region which contains particles flown and also generated particle population to take out the most optimum path. They concluded that the described algorithm is advantageous because of having faster search speed and better search quality. In [6], authors have adopted a PD controller to track the optimal centre path to determine the optimal route at quick convergence along with global search character. Huang, [7] has described FPGA based master-slave PPSO along with B-spline and applied it for the sensor based robot to make the path smoother. Fitness value indicates the approaching behavior for each approach. Attuning of fuzzy logic criterions applying PSO was proposed in [8]. Ahmadzadeh and Ghanavati, [9] have described the problem as optimization problem and found the navigation using PSO algorithm. To reach final goal, local goal was made one of the key points. Masehian and Sedighizadeh, [10] have initiated a new technique for motion planning of wheeled robot. Mutation of PSO and PRM has been done by them in which both techniques have probabilistic elements and parameters. The random nodes generated in the PRM along with the idea of the particles in PSO. They adopted static 2D obstacles with known vertices. In [11] author has described the social acceptation of knowledge of the PSO algorithm. Robinson, et al. [12] have proposed PSO, GA, and their hybrids.

In [13] author has proposed an enhanced PSO algorithm adopting the inertia weight, which reduces in accordance with iterative generation increasing. Quin, et al. [14] have proposed a modified particle swarm optimization approach by inserting a hybridization mechanics which was capable of dodging local minimum. Karimi and Pourtakdoust, [15] have developed a hybrid algorithm using heuristic optimization approach. This optimization algorithm was cable of reducing computational effort for the rapid convergence to an optimal path and dodge obstacle. The approach was beneficial to increase the efficiency of PSO to track dynamic changes with new and efficient features.

\section{Genetic Algorithm Encoded in Wheeled Robot}

Holland, [16] had introduced the concept of Genetic Algorithm. It is a heuristic search approach established on the axioms of the Darwinian concept of endurance of the fittest and natural genetics. Genetic Algorithms are a special class of Evolutionary Algorithms (EA) that utilizes approaches which is eager through evolutionary biology alike as inheritance, mutation, selection, and crossover [17]. Oleiwi and Roth, [18] have considered a complex terrain consists of static distributed obstacles, and figured out a multi-object global route selection challenge for wheeled robot navigation.
Mohanta, et al. [19] have described how a GA could be integrated with petri-net model in such a way that it would be helpful in making an integrated navigational controller. They selected multiple wheeled robots, and it was a big challenge to avoid self-collision. This challenge was efficiently taken care by Petri-GA model. It was more efficient and reliable than simple GA model. Schaffer, [20] has described the application for a multi-objective evolutionary search with regards to evolutionary computation. In [21] Castillo et al. have represented the application of a Genetic Algorithm (GA) for offline point-to-point autonomous wheeled robot path planning challenges. It implies to produce efficient paths or trajectories for a Holonomic Robot which was used to move following a flat map of terrain from a starting position to a goal. A two-dimensional grid was used to portray visually, with dangerous ground and obstacles that the Robot have to dodge.

In [22-24], all focused on the theory of Pareto optimality and the Pareto optimal set. Employing these notions of optimality of individuals computed under a multi-objective problem. Dominance and non-dominance based Pareto optimality fitness assignment of each individual was proposed in a present population during an evolutionary search [25]. A genetic Route outlining approach for wheeled robot, which described the vertices as polygonal clusters, has been introduced in Wang, et al. [26]. And these polygon clusters were encoded as genes; in addition invalid routes were represented to penalty function evaluation. Arora, et al. [27] have used MATLAB to describe path planning using GA and used fitness function as the minimum of distance between the two points $=\sqrt{\left(x-x_{2}\right)^{2}+\left(y-y_{2}\right)^{2}}$ Navarro, et al. [28] have implemented the mutation of GA and NN for wheeled robot route outlining. The GA is implemented to coach the neural network is described in Figure 2.

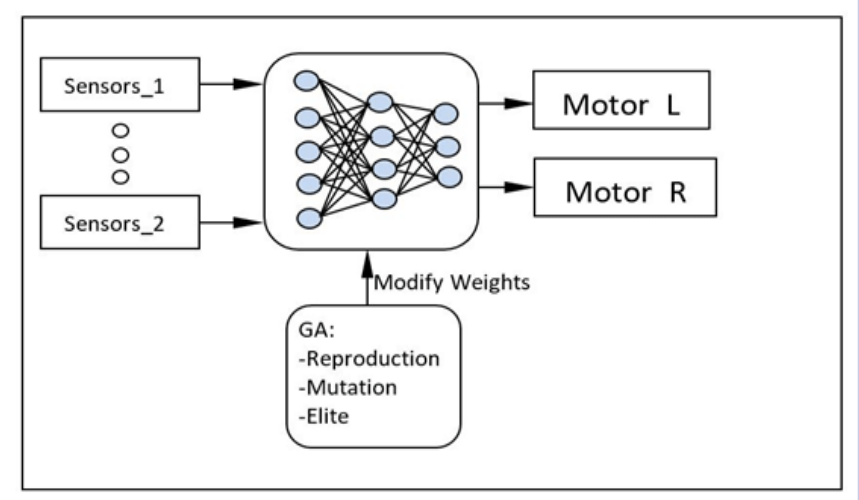

Figure 2: Wheeled robot navigation using mutation of GA and NN algorithms

\section{Fuzzy Logic Approach Encoded in Wheeled Robot}

Determining route and control of a wheeled robot in an unknown and messy terrain are difficult challenge to carry out. Fuzzy logic control is a very important tool for the wheeled robot in the field of selection of route [29]. Abadi and khooban, [30] have used an optimal Mamdani-type fuzzy logic controller for route tracking of wheeled robots (WMRs). Matlab/Simulink 
environment was taken to solve the dynamic model of a nonholonomic wheeled robot. Surmann, et al. [31] selected fuzzy rule-based system to control the movement of an autonomous wheeled robot. Xianhua, et al. [32] have described how fuzzy logic was used for path chasing of a wheeled robot and predictive control. For the prediction of the state and the orientation of the robot, predictive control was implemented. Whereas to handle the nonlinear characteristics of the system the fuzzy control was taken into consideration.

Singh, etal. [33] have measured the robustness of defined fuzzy logic controller by using time necessary for the wheeled robot for simulation and experiment. In [34] Godjevac has described the application of fuzzy logic technique in avoidance of obstacles for wheeled robots. Author has implemented it on a controller based on the Takagi-Sugeno method. The learning procedure was the stochastic approximation method, and the adaptation corresponds to supervised learning of neural networks. A genetic algorithm was introduced by Abdessemed, et al. [35] so that it can extract the rules of a fuzzy controller, planned to control the end effector motion of a planar manipulator in the aim to track a predefined route. In [36] authors have proposed an intelligent fuzzy obstacle avoidance approach for the wheeled robot in non- stable working space consisting of obstacles. They have applied fuzzy logic control for human's driving intelligence and have an advantage of sharp obstacle avoidance and immediate response compared to the traditional model-based method.

Qing-yong, et al. [37] have described the architecture, was sensitive to the sensors uncertainties. He used four designed basic behavior based on priority by incorporating fuzzy logic control. Seraji and Howard, [38] has provided fuzzy logic rules to obtain behavior-based robot navigation and finally obtained the result in the form of the video image with the help of camera attached to board. In Raguraman, et al. [39] has designed a fuzzy logic system with two important points, one of which was dodging obstacle and the second one was recognizing the target. Muthu, et al. [40] has selected proximity sensor to sense obstacles and has described, how robot designed for autonomous navigation to arrive at the target by shunning the hindrances by the implementation of the fuzzy logic controller. The fuzzy logic controller is used by Parhi, [41] for the navigation of multiple wheeled robots. In this model, to doge the hindrances in the unknown environments, the robot controller embedded with fuzzy code (as shown in Figure 3. Petri-Net model) is prepared to dodge impact among one another.

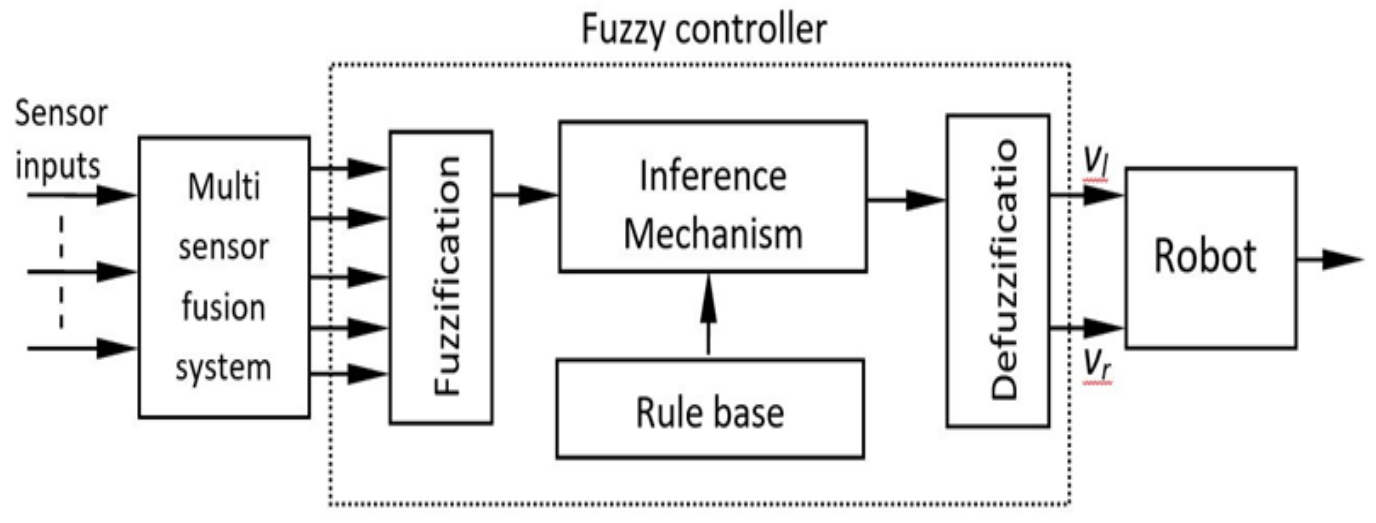

Figure 3: Fuzzy logic algorithm for wheeled robot navigation

\section{Mutation of Fuzzy and Nature-Inspired Algorithm}

Many researchers had already applied fuzzy logic control successfully for several practices. The kernel part of the FLC is a member of linguistic control rules associated by binary approaches of fuzzy inference along with the structural control of interference. Pratihar, et al. [42] have generated a genetic-fuzzy to figure out motion problem in the existence of moving obstacles for the wheeled robot. They used sets of the fuzzy logic controller to navigate between dynamic hindrances, and genetic algorithms were implemented for adjusting the scaling elements of the state quantities. Wu, et al. [43] have used the genetic algorithm with the fuzzy logic controller to drive wheeled robot in pipeline terrain with the help of sensors. They performed simulation and found out that evolution is the key to find the appropriate control mechanism of the fuzzy controller.
Akbarzadeh, [44], authors have described route determining problem over a web of a specify dimension. They used mutation of fuzzy with GA for the location control of a flexible robot link. Besides, they have also used NN-fuzzy for the direct drive motor and Genetic Program (GP)-fuzzy for wheeled robot path planning task. In [45] authors have given a particle swarm optimization algorithm based on evolutionary group to improve fuzzy logic controller for wheeled robot motion planning in undefined environment in 2011. Li, et al. [46] have proposed two motion planning techniques, one of which was the Linear Vertex Decision Mechanism (LVDM) along with the second one was the Fuzzy Logic Decision Mechanism (FLDM). Further, they attached genetic algorithms to the linear vertex decision mechanism so that optimal weight factor would be selected and they also carried genetic algorithm in the FLDM to approach towards the best membership function and/or the number of fuzzy control principle. They have also described computer simulation of the 
evolved linear vertex decision mechanism and fuzzy logic decision mechanism with GA. With the help of their experiment, they have successfully decreases the number of vertices of obstacles. They applied GA to get optimal or sub optimal path.

\section{Neuro-Fuzzy Technique for Wheeled Robot Motion Planning}

A hybrid Fuzzy-Neuro algorithm for wheeled robot motion planning was given by Kundu, et al. [47]. Pradhan, et al. [48] have reviewed about the navigation of multiple wheeled robots by implementing Neuro-Fuzzy code. Total path length and total time taken has been considered to collate with the predefined method with a rule-based algorithm. Hui, et al. [49] have developed a wheeled robot with the help of Neuro-Fuzzy algorithm is like a car. Algabri, et al. [50] have concluded that regarding distance travelled the mutation of PSO and Fuzzy and mutation of Neuro and Fuzzy methods show better performance in comparison to other methods.

$\mathrm{Li},[51]$ has performed simulation and found that navigation in difficult and unknown working space can be efficiently upgraded. He enforced the neuro-fuzzy technique to obtain the behaviorbased control for the robot navigation in non-defined working space. He used two levels, one of them was the high level of environment understanding and the second one was for behavior control. ANFIS is the mutation of Fuzzy logic and neural network. Hegazy, et al. [52] have made it possible for a wheeled robot to arrive the goal in an unknown working space by using the neurofuzzy technique. Lee, [53] has found out the direction for robots to move by inputting the neural network, which was resolute by the interspace and presence of other robots. Fitness function as usual done the most important job, there it described how much group behaviors fit sufficiently with the target. Fitness has been validated the system through simulation, and it was found using a genetic algorithm.

Pulasinghe, et al. [54] have figured out to control machines using spoken language commands using the fuzzy-neural network. Rusu, et al. [55] have experimented with the indoor condition by using infrared and contact sensors to dodge collisions and follow its target. Vatankhah, et al. [56] have matured a Nero-Fuzzy system to reach the target and for behaviour control. AbuBaker, [57] has described how we can get a navigation system by shunning obstacles using the hybrid Neuro-Fuzzy algorithm. He performed many iterations to obtain optimum path by reducing the time and using a broad amount of if-then rule. Baturone, et al. [58] have provided navigation structure using neuro-fuzzy algorithm control system for the wheeled robot at low-cost. They found an efficient result for reaching the goal and dodging obstacles. Wheeled robot navigation on the basis of a neurofuzzy arrangement (displayed in Figure 4) has been described by Li, [59]. A neural network is implemented in this study to find out the reference motion direction, and to control coordinate struggle among the multiple layers of aware action, he used a neural network.

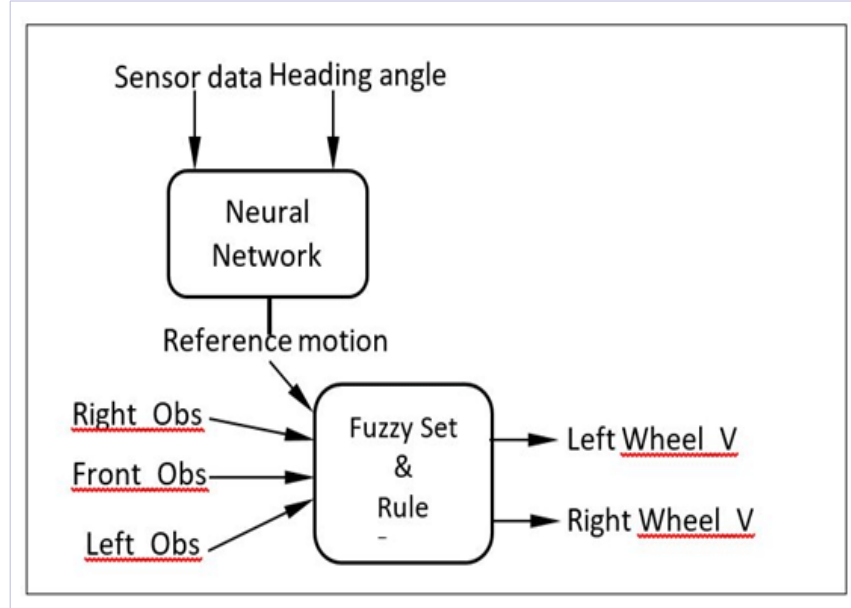

Figure 4: Neuro-Fuzzy algorithm for wheeled robot navigation

\section{Simulated Annealing Algorithm for Wheeled Robot Motion Planning}

Metropolis had introduced SAA in 1953, it is based on the principle of annealing and later Kirkpatrick utilized it for hybrid optimization [60-61]. It was related with global optimization technique that transverses the search space by testing random mutation on an individual solution. Yue and Wang, [62] have applied SAA with compound shape method for the route outlining for the neural network of wheeled robot. Along with achieving the global optimal result which identifies SA technique, it was used also for an ideal declining inclination, thus the convergence value being refined. In Gao and Tian, [63] has evaluated that refined simulated annealing mixed optimize technique and the artificial neural network were implemented to the wheeled robot motion planning. It improved the protective measure and also enhanced the convergence value of simulated annealing algorithm. It also minimizes the computing time of route outlining; thereby it becomes easy and fast for obtaining the global optimal solution.

Janabi-Sharifi and Vinke, [64] has applied simulated annealing technique, whenever robot got trapped in local minima. Authors have applied it for local path planning in a stationary environment, so as to increase the effectiveness of selecting path and to ensure robustness. Statistical mechanics is the base for simulated annealing algorithm and was uplifted by the tempering of metals [65,66]. Miao and Tian, [67] has tested the analytical technique on the basis of simulated annealing algorithm by applying it on the robot for route outlining in the dynamic environments. It is concluded that proposed algorithm has better results in term of processing time in comparison of Dijkstra algorithm. Chang and Song, [68] have described a sensor-based autonomous navigation for wheeled robot in a dynamic environment. Martinez-Alfaro and Gomez-Garcia, [69] has proposed the fuzzy logic and simulated annealing to design an automated route outlining approach of wheeled robot. Proposed algorithm has been used to regulate the velocity of the wheeled robot at the time of navigation, and it was applied in-between of the fixed polygonal hindrances by applying forty-nine fuzzy rules to get collision free optimal path. 
In [70] author has adopted Artificial Potential Field (APF) technique and Simulated Annealing technique to describe global path planning method for the wheeled robot. In [71], the authors have adjusted and optimized the preceding and the subsequent criterion of the fuzzy membership function. They tested it to obtain the solution of the optimization challenges of the servo setups. In [72] authors have presented simulated annealing Algorithm to figure out the off-line path planning challenges for wheeled robot. They optimized the algorithm to enhance the behavior of the described algorithm. Liang and $\mathrm{Xu}$, [73] have proposed a mutated simulated annealing technique to apply it on wheeled robot global navigation. They modified it because simulated annealing Algorithm has a demerit of slow convergence rate. Nakamura and Kehtarnavaz, [74] have used the genetic algorithm and SAA combinatorial optimization techniques for hurdle avoidance and AMR. Miao and Tian, [75] has proposed a smart navigational controller based on simulated annealing algorithm. Searching for an optimal or near-optimal route in the dynamic as well as static environment become easy with the application of proposed controller. Synodinos and Aspragathos, [76] has proposed integration of simulated annealing algorithm and artificial potential field. It restricts the robot from going in unwanted local minima challenge in the course of navigation.

\section{Ant Colony Optimization Algorithm and Other Nondeterministic Approaches Encoded in Wheeled Robot}

ACO Uses many ants to transverse the solution space and find the locally productive area. ACO algorithm is inspired by nature. Dorigo and Gianni, [77] have provided a unitary view and described two paradigmatic applications of ACO algorithms. Zhu, et al. [78] have discussed that Memetic Algorithm (MA) was examined on simulated maps and correlated with other counterpart algorithms. They also described that the result came from memetic algorithm had better efficiency than the other compared algorithms. Botzheim, et al. [79] have described a bacterial MA to recognize a collision-free route for wheeled robot with minimizing the path length and the number of diverging. Ant Colony Optimization was used to determine the presence of intra-class pheromone secreted by other ants to determine the shortest path [80]. An improved ant colony system algorithm has been used by Zeng, et al. [81] which made it possible to find out each local optimal path quickly. But before adopting this algorithm, they used fuzzy logistic description to confirm the fuzzy environment of robot local area. To calculate the shortest possible path in optimum time for the environment having moving obstacles, Liang, et al. [82] have adopted bacteria to find out an optimal path without collision with the hindrances with the help of bacterial foraging algorithm to make bio-inspired route outlining approach for a wheeled robot.

Artificial ant is known as every single agent in this optimization algorithm. It is efficient to solve various verities of problems, which consists of network routing for telecommunication networks and nonlinear function optimization [83]. A memetic algorithm has been proposed by Shahidi, et al. [84] for route outlining by the application of an original result description and gradient-based local search. Lin, et al. [85] has proposed a categorized MA for the route outlining and arrangement control of swarm robots. In [86] author has worked on increasing the efficiency by making it strictly follow the goal, leave the concave path. It failed in finding the valid path. Because of the defect in some previous optimization technique in complex environment like computation cost of algorithms, unable to find approximate optimal solutions, initial feasible solutions etc. Author in [87] have used an optimization algorithm i.e. ACO (Ant Colony optimization) so that the problem for wheeled robot can be solved by getting optimum path in the workspace having many irregular obstacles. Ganganath, et al. [88] have established field-of-view concept so that, it would be possible for others to describe the algorithm and would be recognized with most real-world robots. Ganganath and Cheng, [89] have presented an off-line path planner for which the basic was Ant Colony Optimization (ACO) algorithm (ACO2-Gauss) for wheeled robots. To improve the strength of ACS for global searching authors in [90] have put the focus on two main aspects which were the establishment of the new mechanism for pheromone updating and by using continuous tuning of a setting parameter. As there are many benefits ACO algorithm such as fast convergence and distribution of computing in parallel problem like travelling salesman can be successfully solved by the help of it [91]. It also has some demerits like it easily trapped in local minimum, at the same time it becomes a challenge to find an optimal path [92].

Hsu and Juang, et al. [93] has proposed wall-following wheeled robot with the help of type-2 fuzzy controller (IT2FC) and hybridized it with an ACO algorithm so as to enhance the execution of the controller. The fuzzy controller is used by the authors to describe the navigation technique for two robots (a follower robot and a leader robot) [94]. Hsu and Juang, [95] have made it possible for the wall-trailing wheeled robot to optimize the rule parameters of the fuzzy controller with the help of multi-objective ACO. A scent pervasion (pheromone) principle of ant (ACO) has been proposed by Chen, et al. [96] which was established on robotic path planning in a map environment. To calculate the shortest possible path in optimum time for the environment having moving obstacles Hossain and Ferdous and have described Bacterial Foraging Optimization (BFO) algorithm for wheeled robot motion planning [97]. Brand and $\mathrm{Yu}$, [98] have proposed another technique to compare with ACO for path length which should be collision free and computational cost in 2D static as well as dynamic working space. To execute it the used Firefly Algorithm (Glow-worm swarm optimization). Luo and Chang, [99] have reviewed various paper on multisensor union and incorporation and its utilization in the field of Mechatronics, and presented in their paper. Pandey and Parhi, [100] have developed the hybrid fuzzy controller by combining wind drive optimization algorithm and employed this hybrid fuzzy controller for autonomous wheeled robot navigation and collision avoidance in different static and dynamic environments. In article [101], the authors have designed and implemented a hybrid fuzzy architecture for intelligent navigation and control of a wheeled robot in static and dynamic environments. Patle, et al. [102] have presented the firefly algorithm based path planning 
and obstacle avoidance for wheeled mobile robot in uncertain environment. Almayyahi, et al. [103] have presented two fuzzy inference systems, one for obstacle avoidance and other for target reaching of the unmanned ground vehicle in the dynamic environment. Elbanhawi and Simic, [104] have made a critical review on motion planning of robot.

\section{Discussions and Summary}

This article describes a critical review of motion planning of wheeled robot by using different nature-inspired techniques like evolutionary algorithm and swarm based optimization algorithm. Various techniques applied by researchers for wheeled robot motion planning have been reviewed in this work. It has been found that in the recent decade literature survey using computational intelligence approach to find the solution of path planning problem of the wheeled robot is quite large compare to numerical method. However, to minimize the computational difficulty and find the best optimal solution, the researchers have focused on the nature-inspired techniques. Based on the abovementioned literature survey, following conclusions have been drawn:

* Researchers implemented various navigational techniques till now to govern the gesture of wheeled robot in unknown or partially known environments.

* Natured-inspired techniques have been used for many times by the researchers to find the solution of motion planning problem of the wheeled robot.

\section{References}

1. Kennedy J, Eberhart RC. Particle swarm optimization. In: Proceedings of the international conference on neural networks. 1995;4:1942 1948.

2. Heppner FH, Grenander U. A stochastic nonlinear model for coordinated bird flocks. In: Krasner E (ed) The ubiquity of chaos, AAAS Publications. 1990:233-238.

3. Bergh FV, Engelbrecht AP. A study of particle swarm optimization particle trajectories. Inf Sci. 2006;176(8):937-971.

4. Chen X, Li Y. Smooth Path Planning of a Mobile Robot Using Stochastic Particle Swarm Optimization. Proceedings of the 2006 IEEE International Conference on Mechatronics and Automation. 2006.

5. Tang Y, Li Q, Wang L, Zhang C, Yin Y. An Improved PSO for Path Planning of Mobile Robots and Its Parameters Discussion. 2010 International Conference on Intelligent Control and Information Processing. 2010.

6. Yang M, Li Cz. Path Planning and Tracking for Multi-robot System Based on Improved PSO Algorithm. 2011 International Conference on Mechatronic Science, Electric Engineering and Computer. 2011.

7. Huang HC. FPGA-Based Parallel Metaheuristic PSO Algorithm and Its Application to Global Path Planning for Autonomous Robot Navigation. J Intell Robot Syst. 2014;76(3-4):475-488.

8. Debnath SBC, Shill PC, Murase K. Particle swarm optimization based adaptive strategy for tuning of fuzzy logic controller. International
Journal of Artificial Intelligence \& Applications. 2013;4(1):37-50.

9. Ahmadzadeh S, Ghanavati M. Navigation of Mobile Robot Using the PSO Particle Swarm Optimization. Journal of Academic and Applied Studies. 2012;2(1):32-38.

10. Masehian E, Sedighizadeh D. A Multi-Objective PSO based Algorithm for Robot Path Planning. 2010 IEEE International Conference on Industrial Technology. 2010.

11. Kennedy J. The particle swarm: social adaptation of knowledge. In Proceedings of the IEEE Conference on Evolutionary Computation. 1997:303-308.

12. Robinson J, Sinton S, Rahmat-Samii Y. Particle swarm, genetic algorithm, and their hybrids: optimization of a profiled corrugated horn antenna. In IEEE antennas and propagation society international symposium. 2002;1:314-317.

13. Jiao B, Lian $\mathrm{Z}, \mathrm{Gu} \mathrm{X}$. A dynamic inertia weight particle swarm optimization algorithm. Chaos, Solutions and Fractals. 2008;37(3):698-705.

14. Qin YQ, Sun DB, Li N, Cen YG. Path planning for mobile robot using the particle swarm optimization with mutation operator. In Proceedings of the Third International Conference on Machine Learning and Cybernetics. 2004:26-29.

15. Karimi J, Pourtakdoust SH. Optimal maneuver-based motion planning over terrain and threats using a dynamic hybrid PSO algorithm. Aerospace Science and Technology. 2013;26(1):60-71.

16. Holland JH. Genetic Algorithms and Adaptation. Adaptive control of ill-Defined Systems. 1984;16:317-333.

17. Goldberg DE. Genetic Algorithms in search, optimization \& Machine Learning. Addison-Wesley Longman Publishing Co. 1989.

18. Oleiwi KB, Roth H, Kazem BI. Modified Genetic Algorithm Based on $\mathrm{A}^{*}$ Algorithm of Multi Objective Optimization for Path Planning. Journal of Automation and Control Engineering. 2014;2(4):357-362.

19. Mohanta JC, Parhi DR, Patel SK. Path planning strategy for autonomous mobile robot navigation using Petri-GA optimization. Computers and Electrical Engineering. 2011;37(6):1058-1070.

20. Schaffer JD. Multiple objective optimization with vector evaluated genetic algorithms. Genetic algorithms and their applications. In: Proceedings of the first international conference on genetic algorithms. 1985:93-100.

21. Castillo O, Trujillo L, Melin P. Multiple objective genetic algorithms for path-planning optimization in autonomous mobile robots. Soft Comput. 2007;11(3):269-279.

22. Fonseca CM, Fleming CJ. Genetic algorithms for multi objective optimization: formulation, discussion and generalization. In: 5th international conference genetic algorithms. 1993:416-423.

23. Goldberg DE. Genetic algorithms in search, optimization and machine learning. Addison-Wesley Longman. 1989.

24. Srinivas M, Deb K. Multiobjective optimization using nondominated sorting in genetic algorithms. J Evol Comput. 1994;2(3):221-248.

25. Zitzler E, Thiele L. Multiobjective evolutionary algorithms: a comparative case study and the strength Pareto approach. IEEE Trans Evol Comput. 1999;3(4):257-271. 
26. Wang Y, Mulvaney D, Sillitoe I. Genetic-Based Mobile Robot Path Planning Using Vertex Heuristics. In 2006 IEEE Conference on Cybernetics and Intelligent Systems. 2006:1-6.

27. Arora T, Gigras Y, Arora V. Robotic Path Planning using Genetic Algorithm in Dynamic Environment. International Journal of Computer Applications. 2014;89(11):0975-8887.

28. Navarro N, Muñoz C, Freund W, Arredondo VT. Acquiring Adaptive Behaviors of Mobile Robots Using Genetic Algorithms and Artificial Neural Networks. IEEE Conference on Electronics, Robotics and Automotive Mechanics. 2006:87-91.

29. Algabri M, Mathkour H, Ramdane H, Alsulaiman M. Comparative study of soft computing techniques for mobile robot navigation in an unknown environment. Computers in Human Behavior. 2015;50:4256.

30. Abadi DNM, Khooban MH. Design of optimal Mamdani-type fuzzy controller for nonholonomic wheeled mobile robots. Journal of King Saud University - Engineering Sciences. 2015;27(1):92-100.

31. Surmann H, Huser J, Peters L. A Fuzzy System for Indoor Mobile Robot Navigation. Proceedings of 1995 IEEE International Conference on Fuzzy Systems.1995:83-85.

32. Xianhua J, Yuichi M, Xingquan Z. Predictive fuzzy control for a mobile robot with nonholonomic constraints. Proceedings of IEEE International Conference on Advanced Robotics. 2005:58-63.

33. Singh M, Parhi D, Bhowmik S, Kashyap S. Intelligent controller for mobile robot: Fuzzy logic approach. In Paper presented at the 12th international conference of International Association for Computer Methods and Advances in Geomechanics (IACMAG). 2008:17551762.

34. Godjevac J. A learning procedure for a fuzzy system: application to obstacle avoidance. In Proceedings of the International Symposium on Fuzzy Logic. 1995:142-148.

35. Abdessemed F, Benmahammed KH, Monacelli E. A fuzzy based reactive controller for a non holonomic mobile robot. Robotics Autonomous Syst. 2004;47(1):31-46.

36. Ren L, Wang W, Du Z. A New Fuzzy Intelligent Obstacle Avoidance Control Strategy for Wheeled Mobile Robot. IEEE International Conference on Mechatronics and Automation. 2012:1732-1737.

37. Qing-yong B, Shun-ming L, Wei-yan S, Mu-jin A. A Fuzzy BehaviorBased Architecture for Mobile Robot Navigation in Unknown Environments. International Conference on Artificial Intelligence and Computational Intelligence. 2009:257-261.

38. Seraji H, Howard A. Behavior-Based Robot Navigation on Challenging Terrain: A Fuzzy Logic Approach. IEEE Transactions on Robotics and Automation. 2002;18(3):308-321.

39. Raguraman SM, Tamilselvi D, Shivakumar N. Mobile Robot Navigation Using Fuzzy logic Controller. International conference on control, automation, communication and energy conservation. 2009:1-5.

40. Muthu T, Gloude RT, Swaminathan S, Kumar LS. Fuzzy Logic Controller for Autonomous Navigation. International Conference on Communication and Signal Processing. 2012:81-92.

41. Parhi DR. Navigation of mobile robots using a fuzzy logic controller.
Journal of intelligent and robotic systems. 2005;42(3):253-273.

42. Pratihar DK, Deb K, Ghosh A. A genetic-fuzzy approach for mobile robot navigation among moving obstacles. International Journal of Approximate Reasoning. 1999;20(2):145-172.

43. Wu S, Li Q Zhu E, Xie J, Zhichao G. Fuzzy controller of pipeline Robot Navigation by Genetic Algorithm. Chinese Control and Decision Conference. 2008:904-908.

44. Akbarzadeh TMR, Kumbla K, Tunstel E, Jamshidi M. Soft computing for autonomous robotic systems. Comput Electr Eng. 2000;26(1):532.

45. Juang CF, Chang YC. Evolutionary-group-based particle-swarm optimized fuzzy controller with application to mobile-robot navigation in unknown environments. IEEE Transactions on Fuzzy Systems. 2011;19(2):379-392.

46. Li THS, Chiang MS, Jian SS. Motion Planning of an Autonomous Mobile Robot by Integrating GAS and Fuzzy Logic Control. Ninth IEEE International Conference on Fuzzy Systems. 2000:933-936.

47. Kundu S, Parhi R, Deepak B. Fuzzy-neuro based navigational strategy for mobile robot. International Journal of Scientific \& Engineering Research. 2012;3(6):1-6.

48. Pradhan SK, Parhi DR, Panda AK. Navigation of multiple mobile robots using rule-based-neuro-fuzzy technique. International Journal of Computational Intelligence. 2007;3(2):142-152.

49. Hui NB, Mahendar V, Pratihar DK. Time-optimal, collision-free navigation of a car-like mobile robot using neuro-fuzzy approaches. Fuzzy Sets and Systems. 2006;157(16):2171-2204.

50. Algabri M, Mathkour H, Ramdane H, Alsulaiman M. Comparative study of soft computing techniques for mobile robot navigation in an unknown environment. Computers in Human Behavior. 2015;50:4256.

51. Li W, Ma C, Wahl FM. A neuro-fuzzy system architecture for behaviorbased control of a mobile robot in unknown environments. Fuzzy Sets and Systems. 1997;87(2):133-140.

52. Hegazy OF, Fahmy AA, Refaie OME. An intelligent robot navigation system based on neuro-fuzzy control. In: Lecture Notes in Computer Science. 2004;3157:1017-1018.

53. Lee M. Evolution of behaviors in autonomous robot using artificial neural network and genetic algorithm. Inform Sci. 2003;155(12):43-60.

54. Pulasinghe K, Watanabe K, Izumi K, Kiguchi K. Modular fuzzy-neuro controller driven by spoken language commands. IEEE Trans Syst Man Cybern B Cybern. 2004;34(1):293-302.

55. Rusu P, Petriu EM, Whalen TE, Cornell A, Spoelder HJW. Behaviorbased neuro-fuzzy controller for mobile robot navigation. IEEE Transactions on Instrumentation and Measurement. 2003;52(4):1335-1340.

56. Vatankhah R, Etemadi S, Alasty A, Vossoughi G. Adaptive criticbased neurofuzzy controller in multi-agents: Distributed behavioral control and path tracking. Neurocomputing. 2012;88:24-35.

57. AbuBaker A. A novel mobile robot navigation system using neurofuzzy rule-based optimization technique. Res J Appl Sci Eng Technol. 


\section{2;4(15):2577-2583}

58. Baturone I, Gersnoviez A, Barriga A. Neuro-fuzzy techniques to optimize an FPGA embedded controller for robot navigation. Appl Soft Comput. 2014;21:95-106.

59. Li W. Neuro-fuzzy systems for intelligent robot navigation and control under uncertainty, IEEE International Joint Conference on Fuzzy Systems. 1995;1747-1754.

60. Diaz de Leon S JL, Sossa A JH. Automatic path planning for a mobile robot among obstacles of arbitrary shape. IEEE Trans Syst Man Cybern B Cybern. 1998;28(3):467-472. doi: 10.1109/3477.678654

61. Romeijn HE, Smith RL. Simulated Annealing for Constrained Global Optimization. J Global optimization. 1994;5(2):101-126.

62. Yue H, Wang Z. Path Planning of Mobile Robot Based on Compound Shape and Simulated Annealing Hybrid Algorithm. IEEE International Conference on Robotics and Biomimetics. 2005:186-189.

63. Gao M, Tian J. Path Planning for Mobile Robot Based on Improved Simulated Annealing Artificial Neural Network. Third International Conference on Natural Computation. 2007.

64. Janabi-Sharifi F, Vinke D. Integration of the artificial potential field approach with simulated annealing for robot path planning. Proceedings of 8th IEEE International Symposium on Intelligent Control. 1993:536-541.

65. Metropolis N, Rosenbluth AW, Rosenbluth MN, Teller AH, Teller E. Equation of State Calculations by Fast Computing Machines. The J Chem Phys. 1953;21(6):1087-1092. doi: 10.1063/1.1699114

66. Yanar TA, Akyurek Z. Fuzzy Model Tuning Using Simulated Annealing Expert Systems with Applications. 2011;38(7):8159-8169.

67. Miao H, Tian YC. Dynamic Robot Path Planning using an Enhanced Simulated Annealing Approach. Applied Mathematics and Computation. 2013;222:420-437

68. Chang CC, Song KT. Sensor-Based Motion Planning of a Mobile Robot in a Dynamic Environment. IEEE International Conference on Industrial Electronics, Control, Instrumentation. 1996:766-771.

69. Martinez-Alfaro H, Gomez-Garcia S. Mobile Robot Path Planning and Tracking Using Simulated Annealing and Fuzzy Logic Control. Expert Systems with Applications. 1998;15(3-4):421-429.

70. Zhu Q Yan Y, Xing Z. Robot Path Planning Based on Artificial Potential Field Approach with Simulated Annealing. IEEE Proceedings of the Sixth International Conference on Intelligent Systems Design and Applications (ISDA). 2006:622-627.

71. Precup RE, Radac MB, Dragos CA, Preitl S, Petriu EM. Simulated Annealing Approach to Fuzzy Modeling of Servo Systems. IEEE International Conference on Cybernetics. 2013:267-272.

72. Tavares RS, Martins TC, Tsuzuki MSG. Simulated Annealing with Adaptive Neighbourhood: A Case Study in Off-Line Robot Path Planning. Expert Systems with Applications. 2011;38(4):29512965.

73. Liang Y, Xu L. Global Path Planning for Mobile Robot based Genetic Algorithm and Modified Simulated Annealing Algorithm. In ACM Proceedings of Summit on Genetic and Evolutionary Computation. 2009:303-308.
74. Nakamura E, Kehtarnavaz N. Optimization of Fuzzy Membership Function Parameters. IEEE International Conference on Systems, Man and Cybernetics. 1995:1-6.

75. Miao H, Tian YC. Robot Path Planning in Dynamic Environments using a Simulated Annealing Based Approach. IEEE International Conference on Control, Automation, Robotics and Vision (ICARCV). 2008:1253-1258.

76. Synodinos A, Aspragathos NA. Robot Path Planning of a Mobile Robot using Solid Modeling Techniques on Potential Fields. IEEE International Conference on Mechatronics and Embedded Systems and Applications (MESA). 2010:549-553

77. Dorigo M, Car GD. Ant Colony Optimization: A New Meta-Heuristic. Proceedings of the 1999 Congress on Evolutionary Computation. 1999.

78. Zhu Z, Wang F, He S, Sun Y. Global path planning of mobile robots using a memetic algorithm. International Journal of Systems Science. 2015;46(11):1982-1993

79. Botzheim J, Todav Y, Kubota N. Bacterial Memetic Algorithm for Offline Path Planning of Mobile Robots. Memetic Computing. 2012;4(1):73-86.

80. Hsiao YT, Chnang YT, Chien CC. Ant Colony Optimization for Best Path Planning. IEEE International Symposium on Communications and Information Technology. 2004:109-113.

81. Bi Z, Yimin Y, Yisan X. Mobile Robot Navigation in Unknown Dynamic Environment Based on Ant Colony Algorithm. Global Congress on Intelligent Systems. 2009:98-102.

82. Liang XD, Li LY, Wu JG, Chen HN. Mobile Robot Path Planning based on Adaptive Bacterial Foraging Algorithm. Journal of Central South University. 2013;20(12):3391-3400.

83. Siegwart R, Nourbakhah IR. Introduction to Autonomous Mobile Robots. MIT Press. 2004.

84. Shahidi N, Esmaeilzadeh H, Abdollahi M, Lucas C. Memetic Algorithm Based Path Planning for a Mobile Robot. International Journal of Information Technology. 2004;1(3):100-103.

85. Lin C, Chen K, Chuang W. Motion Planning using a Memetic Evolution Algorithm for Swarm Robots. International Journal of Advanced Robotic Systems. 2012;9(25):1-9.

86. Chen X, Zhao Y, Han J. An improved ACO_based robotics path planning method. J Contr Thory Appl. 2007.

87. Fan X, Luo X, Yi S, Yang S, Zhang H. Optimal Path Planning for Mobile Robots Based on Intensified Ant Colony Optimization Algorithm. IEEE International Conference on Robotics, Intelligent Systems and Signal Processing. 2003:131-136.

88. Ganganath N, Cheng CT, Tse CK. An ACO-based Off Line Path Planner for Nonholonomic Mobile Robots. IEEE International Symposium on Circuits and Systems (ISCAS). 2014:1038-1041.

89. Ganganath N, Cheng CT. A 2-Dimensional ACO-based Path Planner for Off-line Robot Path Planning. International Conference on Cyber-Enabled Distributed Computing and Knowledge Discovery. 2013:302-307. 
90. Hsu CC, Hou RY, Wang WY. Path Planning for Mobile Robots Based on Improved Ant Colony Optimization. IEEE International Conference on Systems, Man, and Cybernetics. 2013:2777-2782.

91. Shaogang Z, Ming L. Path Planning of Inspection Robot Based on Ant Colony Optimization Algorithm. International Conference on Electrical and Control Engineering. 2010:1474- 1477.

92. Yuan M, Sunan W, Li P. A model of ant colony and immune network and its application in path planning. 3rd IEEE Conference on Industrial Electronics and Applications. 2008:102-107.

93. Hsu CH, Juang CF. Evolutionary Robot Wall-Following Control using Type-2 Fuzzy Controller with Species-DE-Activated Continuous ACO. IEEE Transactions on Fuzzy Systems. 2013;21(1):100-112.

94. Juang CF, Lai MG, Zeng WT. Evolutionary Fuzzy Control and Navigation for Two Wheeled Robots Cooperatively Carrying an Object in Unknown Environments. IEEE Trans Cyberns. 2015;45(9):17311743. doi: 10.1109/TCYB.2014.2359966

95. Hsu CH, Juang CF. Multi-Objective Continuous-Ant-ColonyOptimized FC for Robot Wall-Following Control. IEEE Computational Intelligence Magazine. 2013;8(3):28-40.

96. Chen X, Kong Y, Fang X, Wu Q. A Fast Two-Stage ACO Algorithm for Robotic Path Planning. Neural Computing and Applications. 2013;22(2):313-319.

97. Hossain MA, Ferdousand I. Autonomous Robot Path Planning in Dynamic Environment using a New Optimization Technique Inspired by Bacterial Foraging Technique. Robotics and Autonomous Systems. 2015;64:137-141.
98. Brand M, Yu XH. Autonomous Robot Path Optimization using Firefly Algorithm. IEEE International Conference on Machine Learning and Cybernetics (ICMLC). 2013:1028-1032.

99. Luo RC, Chang CC. Multisensor Fusion and Integration: A Review on Approaches and Its Applications in Mechatronics. IEEE Transactions on Industrial Informatics. 2012;8(1):49-60.

100. Pandey A, Parhi DR. Optimum path planning of mobile robot in unknown static and dynamic environments using Fuzzy-Wind Driven Optimization algorithm. Defence Technology. 2017;13(1):4758.

101. Pandey A, Pandey S, Gupta P. Intelligent navigation and control of a mobile robot in static and dynamic environments using hybrid fuzzy architecture. International Journal of Autonomic Computing. 2017;2(3):255-281.

102. Patle K, Pandey A, Jagadeesh A, Parhi DR. Path planning in uncertain environment by using firefly algorithm. Defence Technology. 2018.

103. Almayyahi A, Wang W, Hussein AA, Birch P. Motion control design for unmanned ground vehicle in dynamic environment using intelligent controller. International Journal of Intelligent Computing and Cybernetics. 2017;10(4):530-548.

104. Elbanhawi M, Simic M. Sampling-based robot motion planning: A review. IEEE Access Practical Innovations. 2014;2:56-77. 